\title{
Sigmoid Volvulus After Diagnostic Endoscopy
}

Colonoscopy is a widely used technique for examination of the lower gastrointestinal tract. Complications are unusual, with the most frequent reported being perforation or haemorrhage, usually after polypectomy (1). We report here a rare complication of sigmoid volvulus occurring after diagnostic colonoscopy.

A 73-year-old man was referred for colonoscopy after reporting a change in bowel habit. He had had no previous bowel problems, and a rigid sigmoidoscopy examination to $20 \mathrm{~cm}$ was normal. Standard bowel preparation of a solution containing polyethylene glycol was prescribed 24 hours before the procedure, but due to nausea the patient only managed half the prescribed dose. Consequently, colonoscopy was only possible as far as the splenic flexure, with further insertion limited by semiliquid faeces. No gross abnormality was seen up to this point, although a long, redundant sigmoid was noted.

The patient was discharged home for later review. After 24 hours, he was seen by his primary physician, who noted abdominal distension and generalized pain. He was immediately admitted to hospital, where the clinical assessment and plain abdominal radiograph revealed a large sigmoid volvulus (Figure 1), confirmed by water-soluble enema. This was decompressed with a simple flatus tube. However, the condition recurred two days later during similar bowel preparation for an in-hospital barium enema. An abdominal radiography and soluble contrast enema, as before, confirmed a sigmoid volvulus at the rectosigmoid junction (Figure 2). Surgery revealed a large sigmoid volvulus, adherent to some small-bowel loops. A sigmoid colectomy was carried out successfully, and he was discharged soon after and remains well.

Although it may have been coincidental, the close sequence of events suggests a causal relationship between the onset of the volvulus and either the bowel preparation or the colonoscopy. Certainly, the type of bowel preparation would induce a heavy liquid and solid load in the sigmoid, perhaps making twisting of a mobile redundant colon more likely. Similarly, bowel overinflation in order to insert the colonoscope over the liquid and solid faeces may have initiated an alpha loop in the sigmoid, which subsequently developed into a volvulus. An extensive literature search revealed only one similar previous case, from an American series of over 20,000 procedures (2). Sigmoid volvulus therefore represents a very rare complication of colonoscopy, especially in someone with a long, redundant sigmoid colon.

\section{S. R. Brown, C. S. Orgles, D. J. Lintott, P. J. Finan}

Centre for Digestive Diseases, Leeds General Infirmary, Leeds, United Kingdom

\section{References}

1. Hart R, Classen M. Complications of diagnostic gastrointestinal endoscopy. Endoscopy 1990; 22: 229-33.

2. Smith LE. Fibreoptic colonoscopy: complications of colonoscopy and polypectomy. Dis Colon Rectum 1976: 19: 407-12.

3. Young WS, Engelbrecht HE, Stoker A. Plain film analysis in sigmoid volvulus. Clin Radiol 1978; 29: 553-60.

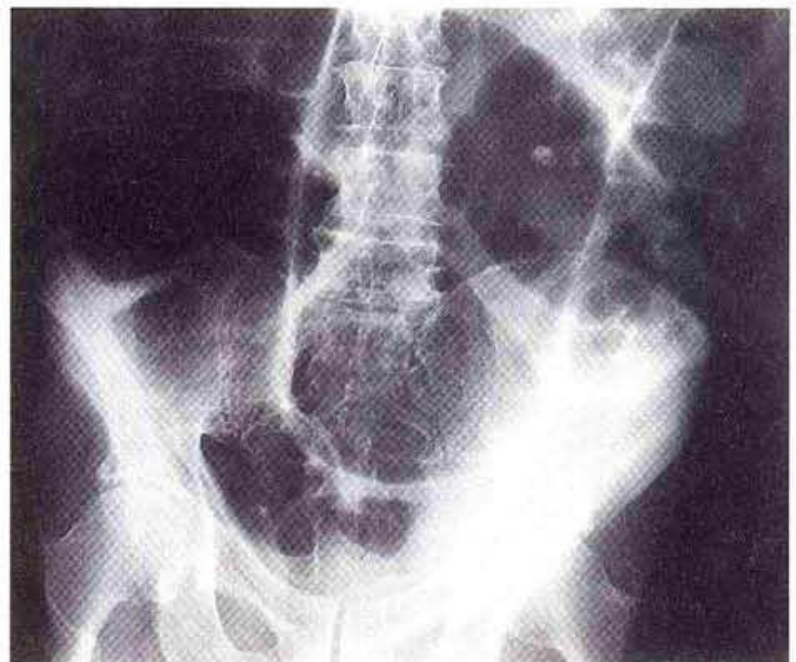

Figure 1: Plain abdominal radiograph, showing a grossly dilated loop of large bowel emerging from the left lower pelvis.

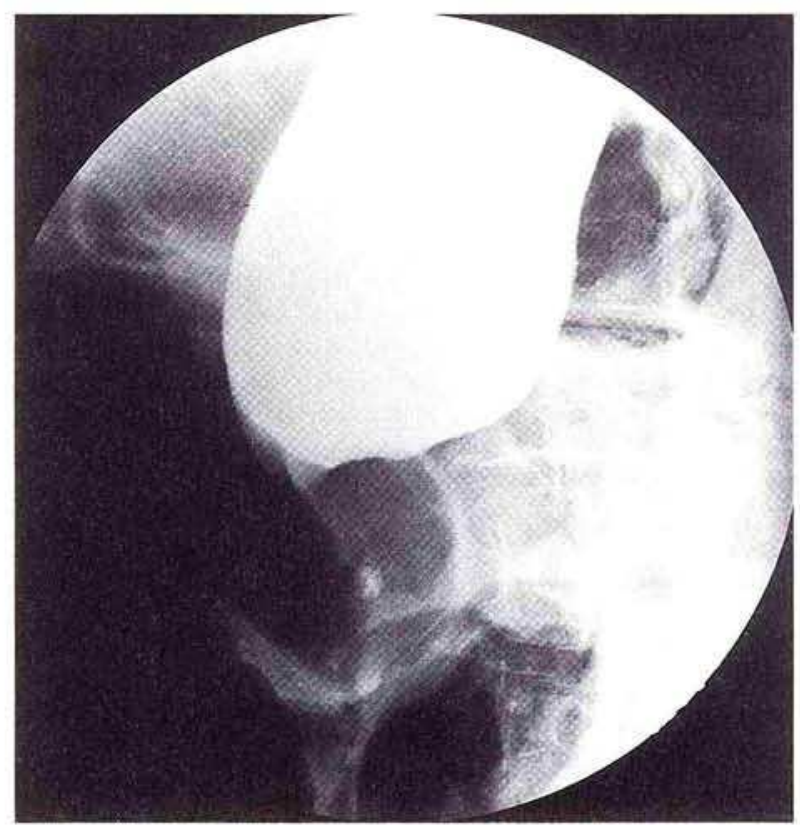

Figure 2: Soluble contrast enema, showing the typical curved, tapered narrowing at the rectosigmoid junction and twisted mucosal folds with a hooked beak appearance (bird-of-prey sign) (3).

Corresponding Author

S. R. Brown

ICRF, Ashley Wing

St. James Hospital

Leeds LS97TF

United Kingdom

Fax: +44-1132-340183 